\title{
Evaluation of Nitric Oxide Scavenging and Nitric Oxide Synthases Expression of Resveratrol and Polydatin
}

\author{
Wei-ding Wang ${ }^{1}$,Zi-qi Lai ${ }^{1}$, Zi-cong Wu ${ }^{1}$,Yi-fei Wang ${ }^{2}$, Zhi-ping Wang ${ }^{1 *}$
}

\author{
${ }^{1}$ Guangdong Pharmaceutical University, School of Pharmacy, Guangdong Provincial Engineering Center of \\ Topical Precise Drug Delivery System, Guangzhou 510006, China \\ ${ }^{2}$ Guangzhou Jinan Biomedical Research and Development center, jinan university, Guangzhou 510632, China \\ *Corresponding author. Email:wzping_jshb@126.com \\ Zi-qi Lai and Wei-ding Wang contributed equally to this study. Wei-ding Wang is a pharmacy grade 17 \\ undergraduate, Zi-qi Lai is a pharmacy (Integrated Pharmacy) grade 17 undergraduate of Guangdong \\ Pharmaceutical University
}

\begin{abstract}
Cellular damage induced by free-radicals like reactive oxygen species(ROS) has been implicated in several diseases. The ROS radicals like alkoxy radical(RO-) and peroxy radical (ROO-) are similar to those that are physiologically active and thus might initiate a cascade of intracellular toxic events leading to DNA damage and cell death. Hence naturally anti-oxidant play a vital role in combating these conditions. Resveratrol (RES) and polydatin (PD) are two kinds of natural phytoalexin with excellent antimicrobial and anti-inflammatory activity. In this study aim to evaluation the antiinflammatory ability of RES and PD on LPS-stimulate inflammatory cell model by Nitric oxide(NO) colorimetric method and NO synthases expression with qRT-PCR method, to provide a basis for the potential application and development in the future. The results showed that RES is significantly stronger than PD in NO synthases expression $(P<0.05)$. At the same time, the comparison of NO production inhibition rate between RES and PD are not significant $(P>0.05)$. RES and PD show their respective anti-inflammatory activity advantages under different indicators, which means we should choose RES or PD according to the different uses.
\end{abstract}

Keywords: Nitric oxide, scavenging, Nitric oxide synthases, polydatin, Resveratrol

\section{INTRODUCTION}

Reactive oxygen species(ROS) plays an important role in regulating various physiological functions of organisms. The inherent biochemical characteristics of reactive oxygen species (ROS) are the basis of the mechanisms necessary for the growth, adaptation or aging of organisms, which urge researchers to make full use of these active chemicals to contribute to medical progress[1]. Thus the demands for free radical scavenging have drawn increasing attention in recent years.

Resveratrol(RES, Fig.1a) (Trans-3,4,5-trihydroxystilbene) is a common plant preservative and is found in some edible substances, such as grape skins, peanuts and red wine. Some people speculate that dietary resveratrol may play an antioxidant role, promote the production of nitric oxide, inhibit platelet aggregation, increase high density lipoprotein cholesterol, and thus play a protective role in the heart[2]. Since then, Res has been shown to exert a variety of pharmacological effects such as antioxidant, antidiabetes, anti-inflammatory and anti-cancer activities. RES is a natural compound currently under investigation due to its important biological anticancer properties, including effects on leukemia, skin, breast, lung gastric, colorectal, neuroblastoma, pancreatic and hepatoma cancers [3-7].

Polydatin or piceid (PD, Fig.1b), resveratrol-3-O- $\beta$ glucopyranoside, also called piceid, is a stilbenoid glucoside of a resveratrol derivative. It derives mainly from the root and rhizome of Polygonum cuspidatum Sieb. et Zucc. Although the role of P. cuspidatum root in angiogenesis has been reported, the active chemical or chemicals responsible for such function is not known[8]. PD has been reported to exhibit promising pharmacological activities including anti-carcino genic[9], antiplatelet aggregation[10], anti-inflammatory[11], antihemorrhagic shock[12], protect against carbon tetrachloride-induced liver Injury[13], ameliorates oxidative stress-related inflammatory responses resulting in renal injury[14], anti-aging[15], ameliorates insulin resistance and hepatic steatosis[16], and anti-oxidation activity[17]. Recently, Some studies have shown that its antioxidant activity is an important mechanism to protect the vulnerable SNC neurodegeneration in some models.These findings strongly indicate the 
potential therapeutic potential of piceid in the treatment of PD[18].

However, up to now, it is still unclear whether Res and PD has Nitric oxide(NO) scavenging and Nitric oxide synthases expression. In this study aim to evaluation the anti-inflammatory ability of RES and PD on LPS-stimulate inflammatory cell model by NO colorimetric method and NO synthases expression with qRT-PCR method, to provide a basis for the potential application and development in the future.<smiles>Oc1ccc(C=Cc2cc(O)cc(O)c2)cc1</smiles>

a

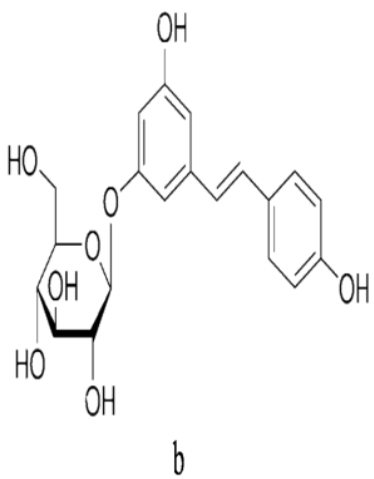

b
Fig.1 Chemical structure of RES (a) and PD (b)

\section{MATERIALS AND METHODS}

\subsection{Materials}

Sodium dihydrogen phosphate, disodium hydrogen phosphate, sodium phosphate, potassium persulfate and MTT were all in analytical grade and obtained from Aladdin Industrial Corporation, China. NO detection kit was from Beyotime, China. PCR Mastermix and cDNA reverse transcriptase kit was from Takara, Japan. TRIzol reagent was from Tiangen, China. Gene specific primers were from Sangon, China. Res and polydatin ( $\geq 98 \%$ by HPLC) were purchased from Aladdin Industrial Inc.(Shanghai, China).

\subsection{Cell culture maintenance}

RAW murine macrophage 264.7 cell line was purchased from Insitute of Biochemistry and Cell Biology, CAS, Shanghai, China. The cells were maintained in Dulbecco's modified eagle's medium (DMEM) supplemented with $10 \%$ fetal bovine serum (FBS), $1 \times$ antibiotic solution [penicillin $(100 \mathrm{U} / \mathrm{ml})$ and streptomycin $(100 \mathrm{U} / \mathrm{ml})]$ in a humidified atmosphere supplied with 5\% $\mathrm{CO} 2$ and maintained at a temperature of $37{ }^{\circ} \mathrm{C}$. Cells were allowed to grow till it reached a confluency of $80-90 \%$ and was washed with phosphate buffered saline (PBS) with regular replacement of culture medium

\subsection{Cell viability}

MTT assay. In brief, RAW 264.7 macrophage cells were seeded in 96-well culture plate. After $24 \mathrm{~h}$ of incubation, the cells were stimulated with varying concentrations of RES and PD (10.0, 25.0, 50.0, 75.0, $100.0,150.0$ and $200.0 \mu \mathrm{M})$ in FBS free DMEM medium for further $24 \mathrm{~h}$. The mother liquor of RSE $(10.0 \mathrm{mM})$ and PD $(10.0 \mathrm{mM})$ were use DMSO as solvent and adding the corresponding volume of DMSO to ensure that the solvent quantity of the systems are equal. After incubation, $10.0 \mu \mathrm{L}$ MTT DMSO solution $(5 \mathrm{mg} / \mathrm{mL})$ was added to each well and the plate was incubated for $4 \mathrm{~h}$ at $37^{\circ} \mathrm{C}$ in dark. Then, remove $90 \mu \mathrm{L}$ medium from each well and 100 $\mu \mathrm{L}$ of cell culture grade DMSO was added to the wells and incubated for 15 min under gentle shaking at 37 ${ }^{\circ} \mathrm{C}$ to dissolve the tetrazolium dye. Relative cell viability was calculated based on the absorbance at $570 \mathrm{~nm}$ and reported as relative percentage viability. Relative cell percentage viability was calculated by following equation:

$$
\text { Cell viability }(\%)=\left[\frac{A_{0}-A_{1}}{A_{0}}\right] \times 100 \%
$$

where A0 is the absorbance of the control and A1 is the absorbance of samples.

\subsection{Treatment of RAW 264.7 macrophages}

Cells reaching a concentration of $5 \times 106$ cells/well were considered for drug treatment. RAW 264.7 macrophages were treated with varying concentrations of RES or PD (10.0, 20.0, 40.0 and $50.0 \mu \mathrm{M})$ and LPS $(100 \mathrm{ng} / \mathrm{mL})$ for $24 \mathrm{~h}$, and the same concentrations of and ascorbic acid and dexamethasone (DEX) were used as standard. The mother liquor of ascorbic acid $(10.0 \mathrm{mM})$ and DEX $(10.0 \mathrm{mM})$ were use DMSO as solvent and adding the corresponding volume of DMSO to ensure that the solvent quantity of the systems are equal.

\subsection{Quantification of mRNA levels by quantitative real-time PCR}

Total RNA was isolated from pre-treated RAW 264.7 macrophage cells with various concentrations of RES, PD, ascorbic acid or DEX (10.0, 20.0, 40.0 and 50.0 $\mu \mathrm{M})$ with LPS (100ng/mL) stimulation for $24 \mathrm{~h}$ using the TRIzol reagent according to manufacturer's instructions. The total RNA was reverse transcribed by using high capacity cDNA reverse transcriptase kit (Takara, Japan) and the mRNA expression was amplified by PCR mastermix. Gene specific primers were designed manually using online NCBI primerBLAST tool. Primer sequences of forward and reverse were at tab.1. Quantitative Real time PCR (qRT-PCR) was performed to measure the gene expression of associated anti-oxidant responsive elements (iNOS) and GAPDH respectively. Transcription levels were assessed utilizing the step one real-time thermal cycler (BioRad, USA) with Takara PCR Master mix according to the instructions of the manufacturer. Thermal cycling conditions were as follows: Denaturation at $95{ }^{\circ} \mathrm{C}$ for $15 \mathrm{~s}$, annealing at $60^{\circ} \mathrm{C}$ for $30 \mathrm{~s}$ and extension at $72{ }^{\circ} \mathrm{C}$ for $30 \mathrm{~s}$. The levels of GAPDH gene expression served as an internal control. 
Tab.1 Sequences of PCR primers used for qRT-PCR.

\begin{tabular}{ccc}
\hline Gene & Primer & Sequence \\
\hline \multirow{2}{*}{ iNOS } & Forward & AAGCAGCTGGCCAATGAG \\
& Reverse & CCCCATAGGAAAAGACTGCA \\
GAP & Forward & GTCATTGAGAGCAATGCCAG \\
DH & Reverse & GTGTTCCTACCCCCAATGTG \\
\hline
\end{tabular}

\subsection{Inhibition of NO production in LPS- stimulated RAW 264.7 cells}

NO production was determined by measuring the level of nitrite in the culture supernatant of RAW 264.7 cells. The RAW264.7 cells were seeded at a density of $5 \times 10^{5}$ cells/well in 96 well plates for $24 \mathrm{~h}$ at $37{ }^{\circ} \mathrm{C}$ and $5 \% \mathrm{CO}_{2}$. Then, the cells were treated with different concentrations of RES (10.0, 20.0, 40.0 and 50.0 $\mu \mathrm{M})$ or PD $(10.0,20.0,40.0$ and $50.0 \mu \mathrm{M})$ with LPS $(100 \mathrm{ng} / \mathrm{mL})$, and the same concentrations of DEX used as standard, prepared in FBS-free DMEM. The presence of nitrite was determined in cell culture media using commercial NO detection kit.

\subsection{Statistical analysis}

Results were expressed as mean \pm standard deviation (SD). Student's t-test was used to compare the mean differences between samples using the statistical software SPSS version 19.0 (SPSS, Chicago). In all cases $\mathrm{P}<0.05$ was considered statistically significant.

\section{RESULTS AND DISCUSSION}

\subsection{Cell viability}

To determine the cytotoxic concentrations of RES and PD, MTT assay was evaluated (Fig. 2). The viability of RAW 264.7 cells was not significantly decreased by 24 incubation with up to $50 \mu \mathrm{M}$ (cell viability > $90 \%$ ) of RES and PD, the IC50 value of RES is $100.1 \pm 2.1 \mu \mathrm{M}$ and the IC50 value of PD is higher than $200.0 \mu \mathrm{M}$. Based on these results, the concentrations of RES and PD, $10.0 \mu \mathrm{M}, 20.0 \mu \mathrm{M}, 40.0 \mu \mathrm{M}$ and 50.0 $\mu \mathrm{M}$ were selected for further experiments.

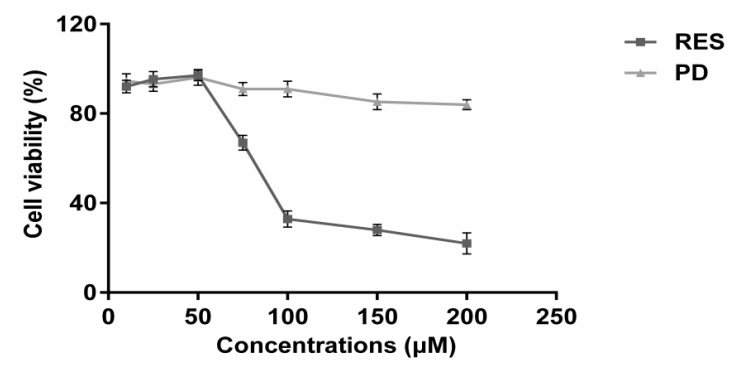

Fig.2 Cell viability of RES and PD at different concentrations (10.0, 25.0, 50.0, 75.0, 100.0, 150.0 and $200.0 \mu \mathrm{M})$

\subsection{Quantification of $m R N A$ levels by real- time PCR}

The un-stimulated RAW 264.7 cells expressed very low mRNA levels of iNOS. Whereas mRNA expressions were strongly expressed after the RAW 264.7 cells were stimulated with LPS $(100 \mathrm{ng} / \mathrm{mL})$. However, treatments with RES or PD significantly (P $<0.05)$ suppressed the expression of iNOS stimulated by LPS $(100 \mathrm{ng} / \mathrm{mL})$. Both RES and PD effectively suppressed the expression of iNOS $(70.67 \%-81.00 \%)$ at the corresponding concentration (Fig.3a). The IC50 values of DEX, RES and PD are 43.5 $\pm 2.6,18.8 \pm 4.0$, $118.6 \pm 3.6 \mathrm{nM}$ respectively, and the inhibition effect of ascorbic acid is too weak to calculate the IC50 value. According to the data, the ability of iNOS expression inhibition of RES is significantly stronger than that of $\mathrm{PD}(\mathrm{P}<0.05)$ and DEX $(\mathrm{P}<0.05)$, and PD is weaker than DEX.
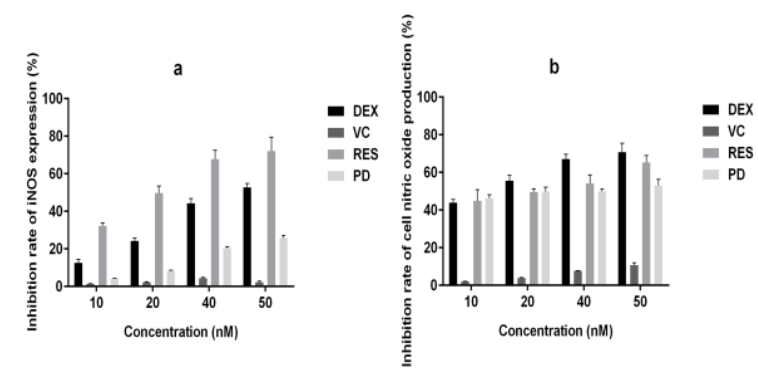

Figure.3. RES and PD on iNOS expression (a) and inhibition rate of NO production (b). The macrophage RAW 264.7 cells were treated with different concentrations of RES and PD (10.0, 20.0, 40.0 and $50.0 \mu \mathrm{M})$ to determine the expression and NO production of stimulated by LPS, and the same concentrations of ascorbic acid and DEX were used as standard.

\subsection{Inhibition of NO production in LPS- stimulated RAW 264.7 cells}

The inhibition effect of NO production by LPSstimulated RAW 264.7 cells was measured by Griess reagent method. When compared to LPS control, both RES and PD treated cell released a lower level of NO in the culture medium, both RES and PD significantly inhibited $(\mathrm{P}<0.05)$ the nitrite accumulation in LPSstimulated RAW264.7 cells in a concentration dependent manner (Fig.3b), suggesting both RES and PD are NO inhibitory components. Among the RES and PD. RES and PD significantly reduced over $60 \%$ of nitrite at their maximum concentrations. The IC50 value of RES $(21.5 \pm 3.2 \mu \mathrm{M})$ is lower than the PD $(23.7 \pm 4.9 \mu \mathrm{M})$ but not significantly $(\mathrm{P}>0.05)$, and the IC50 value of RES is higher than DEX $(15.8 \pm 2.8 \mu \mathrm{M})$ but still not significantly $(\mathrm{P}>0.05)$. At the same time, the IC50 value of PD is significantly higher than DEX (P > 0.05), and the inhibition effect of ascorbic acid is too weak to calculate the IC50 value. 


\subsection{Discussion}

The LPS-stimulated macrophages up-regulate the expression of iNOS, which ultimately results in uncontrolled production of NO. We also performed quantitative RT-PCR to determine whether RES and PD can suppressed the expression of iNOS. The expression of iNOS is related to oxidative stress, and is also related to NF-KB signaling pathway[19]. Ascorbic acid is a widely used medicine treating oxidative stress, but it has little effect on the NF-KB signaling pathway, which is one of the important pathway iNOS expression activated by LPS stimulation[20]. It has been proved by our results. Next, we employ DEX as a positive drug in the cell systems because it's a widely used immune regulatory drug in the clinics and it inhibits the iNOS expression and NO production[21].

Excessive production of $\mathrm{NO}$ and other mediators has been concerned in the development of many diseases, such as arthritis, asthma, multiple sclerosis, inflammatory bowel disease, and atherosclerosis. NO is a signaling molecule involved in inflammation via iNOS up-regulation [22,23]. The inhibitory activity of RES and PD on NO production may be due to the suppression of iNOS expression. Interestingly, RES has a stronger inhibition of iNOS expression than DEX, but DEX is more effective in inhibiting the formation of $\mathrm{NO}$, it may be due to the NO generation dose not entirely dependent on the iNOS expression, but also relate to nNOS or eNOS, RES may have a specific inhibitory effect on iNOS but not inhibit the nNOS or eNOS, which requires more research to confirm.

\section{CONCLUSIONS}

The results showed that RES is significantly stronger than PD in NO synthases expression $(\mathrm{P}<0.05)$. At the same time, the comparison of $\mathrm{NO}$ production inhibition rate between RES and PD are not significant $(P>0.05)$. RES and PD show their respective anti-inflammatory activity advantages under different indicators, which means we should choose RES or PD according to the different uses.

\section{ACKNOWLEDGMENT}

This achievement is supported by the talent training project of guangdong province joint training demonstration base for graduate students, the innovation strong school project of department of education of guangdong province, the innovation and entrepreneurship training program for university students, guangdong pharmaceutical university (S202010573044), guangdong provincial department of education university characteristic innovation project(2020KTSCX060).

\section{REFERENCES}

[1] P. Xu; N. Na; S. Gao; C. Geng, Determination of sodium alginate in algae by near-infrared spectroscopy, Desalination and Water Treatment, 168(2019)117-122.

[2] Mattison JA,Wang M,Bernier M,et al.,Resveratrol prevents high fat/sucrose diet-induced central arterial wall inflammation and stiffening in nonhuman primates, Cell Metab, 20(1)(2014) DOI: https://doi.org/10.1016/j.cmet.2014.04.018

[3] A.Bishayee,T.Politis,A.S.,Darvesh,Resveratrol in the chemoprevention and treatment of hepatocellular carcinoma,Cancer.Treat.Rev. 36(2010) 43-53 DOI: https://doi.org/10.1016/j.ctrv.2009.10.002

[4] L.G.Carter,J.A.Dorazio,K.J.Pearson,Resveratrol and cancer:a focus on in vivo evidence, Endocr. Relat. Cancer. 21(2014) R209-R221 DOI: https://doi.org/10.1530/ERC-13-0171

[5] S.Schuster,M.Penke,T.Gorski,et al.,Resveratrol Differentially Regulates NAMPT and SIRT1 in Hepatocarcinoma Cells and Primary Human Hepatocytes, PLoS. One. 9(2014) e91045 DOI: https://doi.org/10.1186/2049-3002-2-S1-P65

[6] H. Zhang,R. Yang,Resveratrol inhibits VEGF gene expression and proliferation of hepatocarcinoma cells, Hepato-gastroenterol. 61(2014) 410-412 DOI: https://doi.org/10.5754/hge11323

[7] G. Regev-Shoshani,O. Shoseyov,I. Bilkis,et al., Glycosylation of resveratrol protects it from enzymic oxidation,Biochem J. 374 (2003) 157-163 DOI: https://doi.org/10.1042/BJ20030141

[8] Wei-Hui H,Huai-You W,Xiang-Peng K,et al.,Polydatin suppresses VEGF-induced angiogenesis through binding with VEGF and inhibiting its receptor signalling, The FASEB Journal, 33(2018, ) DOI: https://doi.org/10.1096/fj.201800750R

[9] Y.S.Zhang,Z.X.Zhuang,Q.H.Meng,et al.,Polydatin inhibits growth of lung cancer cells by inducing apoptosis and causing cell cycle arrest, Oncl. Lett. (7)(2014) 295-301 DOI: https://doi.org/10.3892/ol.2013.1696

[10]F.Orsini,F.Pelizzoni, L.Verotta, et al., Isolation, synthesis, and antiplatelet aggregation activity of resveratrol 3-O-beta-Dglucopyranoside and related compounds, J Nat Prod. 60(1997)10821087 DOI: https://doi.org/10.1021/np970069t

[11] T.Lou,W.J.Jiang,D.Xu,et al.,Inhibitory effects of polydatin on lipopolysaccharide-stimulated RAW 264.7 cells, Inflammation.38(2015)1-8 DOI:10.1007/s10753-014-0087-8

[12]X.M.Wang,R.Song,Y.Y.Chen,et al., Polydatina new mitochondria protector for acute severe hemorrhagic shock treatment, Expert Opin Investig 
Drugs. 22(2013)169-179 DOI: https://doi.org/10.1517/13543784. 2013.748033

[13]H.Zhang,C.H.Yu,Y.P.Jiang, et al.,Protective Effects of Polydatin from Polygonum cuspidatum against Carbon Tetrachloride-Induced Liver Injury in Mice, PLoS ONE. 7 (2012), e46574 DOI: https://doi.org/10.1371/journal.pone.0046574

[14]L.Y.Chen,Z. Lan,Q.X. Lin,et al.,Polydatin ameliorates renal injury by attenuating oxidative stress-related inflammatory responses in fructoseinduced urate nephropathic mice,Food Chem Toxicol. 52(2013) 28-35 DOI: https://doi.org/10.1016/j.fct.2012. 10.037

[15]H.Wen,X.H.Gao,J.H.Qin,Probing the antiaging role of polydatin in caenorhabditis elegans on a chip, Integr Biol. 6 (2014) 35-43 DOI: https://doi.org/10.1039/c3ib40191j

[16]Q. Zhang, Y.Y. Tan, N. Zhang, et al, Polydatin supplementation ameliorates diet-induced development of insulin resistance and hepatic steatosis in rats, Mol Med Rep.11 (2015) 603-610 DOI: https://doi.org/10.3892/mmr.2014.2708

[17]D. Su,Y. Cheng,M. Liu, et al.,Comparision of piceid and resveratrol in antioxidation and antiproliferation activities in vitro,PLoS ONE. 8(2013) e54505 DOI: https://doi.org/10.1371/journal.pone.0054 505

[18] Y.P.Chen,D.Q.Zhang,Z.Liao,et al., Antioxidant polydatin (piceid) protects against substantia nigral motor degeneration in multiple rodent models of Parkinson's disease,Molecular Neurodegeneration, 10(1)( 2015) DOI: https://doi.org/10.1186/17501326-10-4

[19]Z.Y.Gong,Z.Q.Yuan,Z.W.Dong, et al.,Glutamine with probiotics attenuates intestinal inflammation and oxidative stress in a rat burn injury model through altered iNOS gene aberrant methylation,Am J Transl Res. 9(2017) 2535.

[20] S.H.Kwon,S.X.Ma,S.I.Hong, et al.,Lonicera japonica THUNB. Extract Inhibits Lipopolysaccharide-Stimulated Inflammatory Responses by Suppressing NF- $\mathrm{K}$ B Signaling in BV-2 Microglial Cells,J Med Food. 18(2015) 762775 DOI: https://doi.org/10.1089/jmf.2014.3341

[21]B.S.Juliana,V.Lorena,F.C.G.Maria,et al.,AntiInflammatory Effect of Dexamethasone Controlled Released From Anterior Suprachoroidal Polyurethane Implants on Endotoxin-Induced Uveitis in Rats,Invest Opth Vis Sci.57(2016)16711679 DOI: https://doi.org/10.1167/iovs.15-18127

[22] Dai Z, Wu Z,Yang Y,et al.,Nitric oxide and energy metabolism in mammals, Biofactors, 39(4) (2013) DOI: https://doi.org/10.1002/biof.1099

[23] M.J.Rebelo, C. Sousa, P.Valento,et al.,Phenolic profile of Douro wines and evaluation of their NO scavenging capacity in LPS-stimulated RAW 264.7 macrophages,Food. Chem. 163 (2014):
16-22DOI: https://doi.org/10.1016/j.food chem .2014 .04 .070 